Historic, Archive Document

Do not assume content reflects current scientific knowledge, policies, or practices. 



\section{SEED LIST}

A list of forage plant seeds and the incidental seeds commonly found with them.

Many synonyms and secondary common names are included in the list. These unapproved names appear as underscored words. The approved cormon names are supplied through the courtesy of

Dr. Frederick V. Coville, Division of Plant

Exploration and Introduction.

Division of Seed Investigations Bureau of Plant Industry

United States Department of Africulture Weshington, D. C. 1935 
United States

Department of

Agrieulture

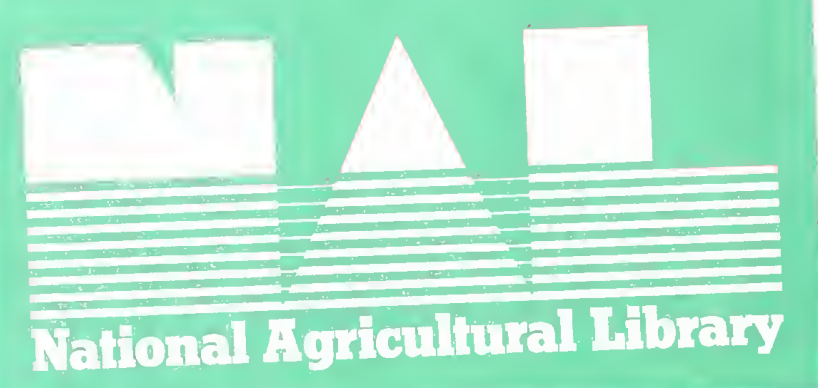




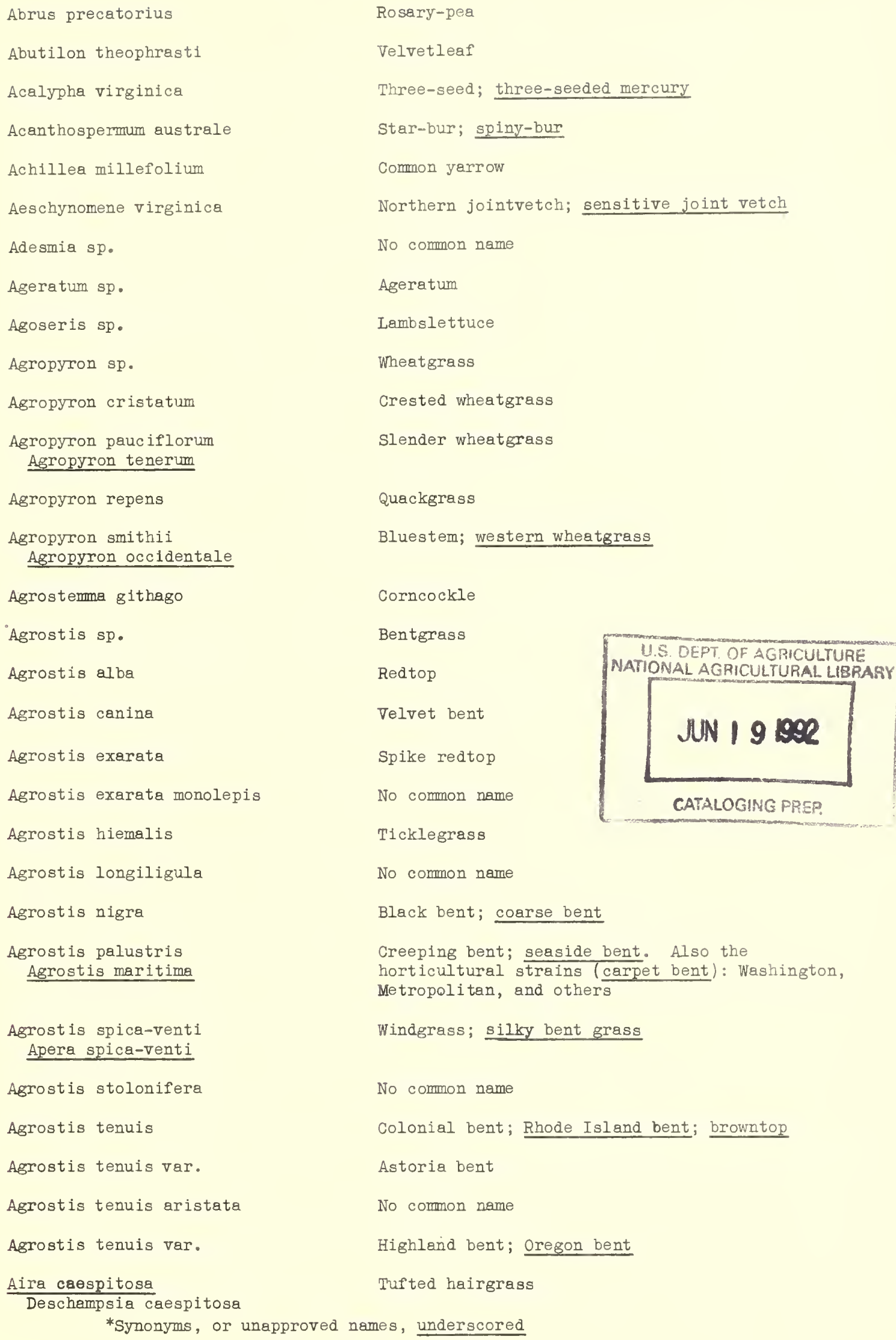


wet 48 


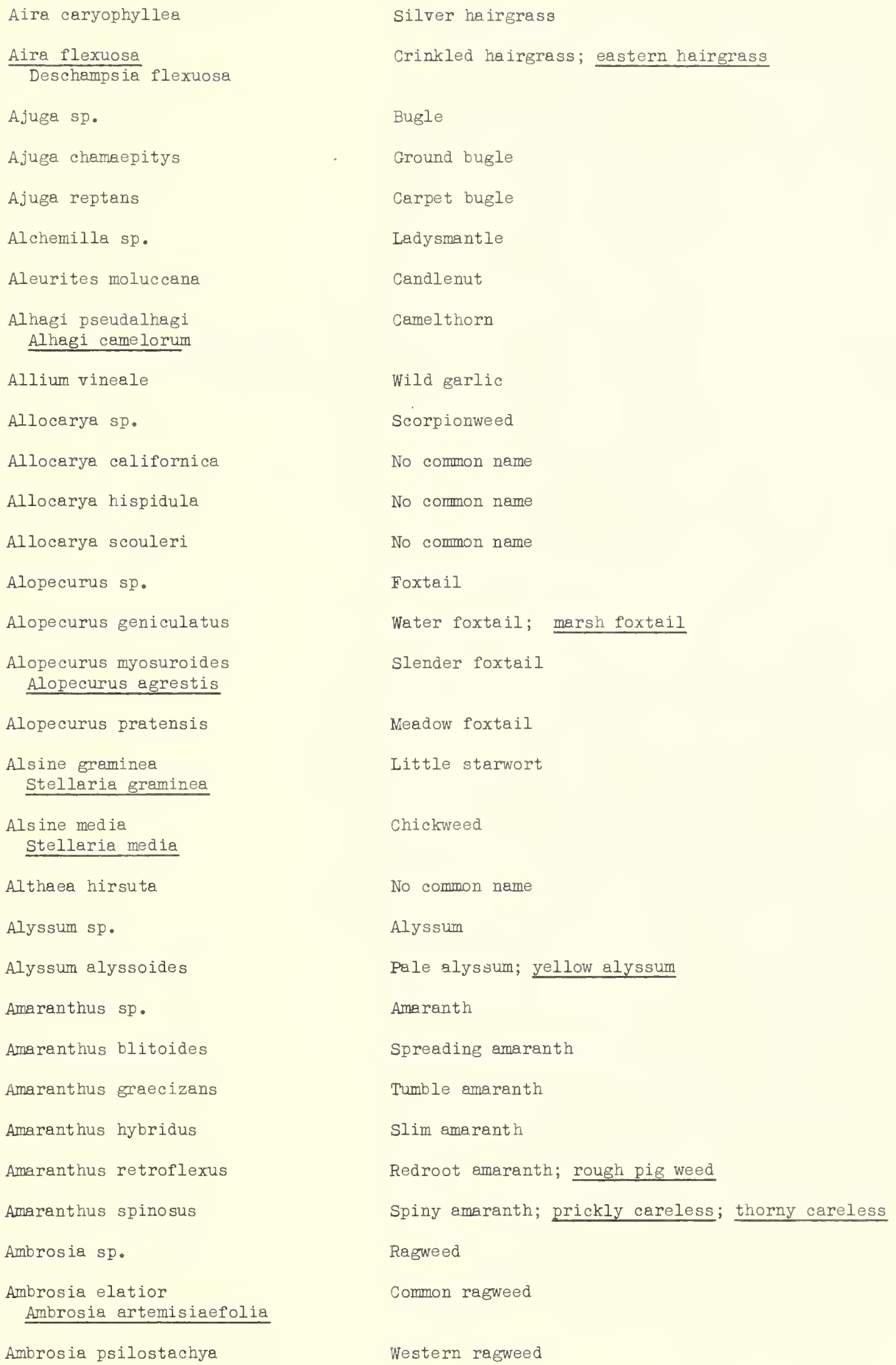




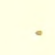




$$
\text { Ambrosia trifida }
$$

Ammi visnaga

Amsinckis sp.

Amsinckis intermedis

Amorphs canescens

Anagallis arvensis

Andropogon sp.

Andropogon glomeratus

Andropogon sorghum sudanens is Holcus sorghum sudanensis Sorghum vulgare sudanense

Andropogon virginicus

Anthemis arvensis

Anthemis cotula

Anthemis tinctoria

Anthoxanthum sp.

Anthoxanthum aristatum Anthoxanthum puelii

Anthoxanthum odoratum

Anthriscus sylvestris

Anthyllis vulneraria

Antirrhinum sp.

Antirrhinum orontium

Apargis sp.

Apargis sutumnalis

Leontodon sutumnalis

Apargia nudicaulis

Leontodon nudicaulis

Apera spica-venti Agrostis spica-venti

\section{Apium armi}

Apocynum sp.

Apocynum cannabinum

Arabidopsis thaliana Stenophragma thaliana

Arsbis sp.

Arabis glabra
Big ragweed; great ragweed

Toothpickplant

Fiddleneck

Cosst fiddleneck

Leadplant

Red pimpernel

Beardgrass

Bushy beardgrass

Sudan grass

Broomsedge

Field camomile

Mayweed

Yellow camomile

Vernalgrass

Annual vernalgrass

Sweet vernalgrass

wild chervil

Kidneyvetch

Snapdragon

Corn snapdragon

No cormon name

Fall-dandelion

Rough hawkbit

Windgrass; silky bentgrass

No common name

Dogbane

Hemp dogbane; Indian-hemp

Mouse-ear-cress

Rockcress

Towermustard 


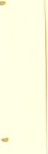


Arctium sp.

Arctium lappa

Arenaria sp.

Arenaria serpyllifolia

Argemone sp.

Argemone alba

Argemone hispida

Aristida sp.

Aristida dichotoma

Aristida stricta

Arnoseris minima

Arrhenatherum elatius

Artemisia sp.

Artemisia annug

Artemisia biennis

Asphodelus tenuifolius

Aster ericoides

Aster frondosus

Aster oblongifolius

Astragalus $\mathrm{sp}$.

Astragalus sinicus

Atriplex sp.

Atriplex hastata

Atriplex hortensis

Atriplex rosea

Atriplex semibaccata

Atriplex truncata

Avene fatua

Avena flavescens

Tribetum flavescens

Avena nuda

Avena pubescens

Avene sativa
Burdock

Big burdock

Sendwort

Thymeleaf sandwort

Pricklepoppy

White pricklepoppy

Hedgehog pricklepoppy

Three-awn

Poverty three-awn

Pineland three-awn

Lemb-succory

Tall oatgrass

Wormwood

Sweet wormwood

Biennial wormwood

No cormon neme

Heath aster

No common name

Aromatic aster

Astragalus

Genge

Saltbush

Fat-hen; saltbush

Garden orach

Redscale; red orach

Australian saltbush

Wedgescale orach; saltbush

Wild oat

Yellow trisetum; yellow oatgrass

Naked oat

Perennial oat; downy oatgrass

oat 

Axonopus compressus

Paspalum compressum

Axyris amaranthoides

Barbarea barbarea Barbarea vulgaris Campe barbarea

Barbarea verna Barbarea praecox Cempe verne

Bassia sp.

Bassia hyssopifolia Kochia hyssopifolia

Beckmannia sp.

Beckmannia syzigachne

Bellis perennis

Berteroa incana

Bidens bipinnata

Bidens cernua

Bidens frondosa

Bignonia radicans Tecoma radicans

Boebera papposa Dyssodia papposa

Boerhaavia erecta

Boisduvalia densiflora

Boisduvalia stricta

Brassica sp.

Brassica adpressa

Brassica alba Sinapis alba

Brassica arvensis Sinapis arvensis

Brassica campestris

Brassica juncea

Brassica napus

Brassica napus var.

Brassica nigra

Brassica oleracea var.

Brassica rapa var.
Carpet grass

Russian $\propto$ pigweed

Bitter wintercress

Early wintercress

Bassia

Fivehook bassia

Sloughgrass

American sloughgrass

English daisy

Hoary alyssum

Spanish-needles

Nodding sticktight; smaller bur-marigold

Sticktight; beggar-ticks

Trumpetcreeper

Prairie-dog-weed; false dog-fennel

Spiderling

No common name

No common name

Mustard

No common name

White mustard

Charlock

Bird rape; wild kale; wild turnip; common yellow mustard

India mustard; brown mustard

Winter rape

Oilseed rape; annual oilseed rape

Black mustard; trieste brown mustard

Cabbage, cauliflower, broccoli, brussels sprouts, kale, collards

Turnip rape; biennial turnip rape 


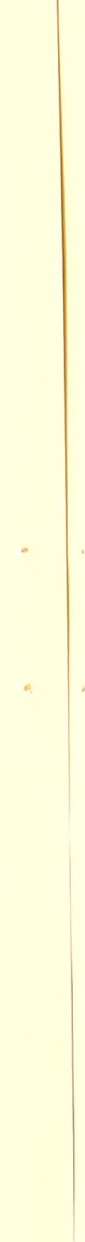


Briza media

Briza minor

Bromus sp.

Bromus arvensis

Bromus brizaeformis

Bromus carinatus

Bromus marginatus

Bromus catharticus Bromus unioloides

Bromus commutatus Bromus pratensis

Bromus hordesceus Bromus mollis

Bromus inermis

Bromus racemosus

Bromus rigidus Bromus maximus

Bromus secalinus

Bromus sterilis

Bromus tectorum

Bromus unioloides Bromus catharticus

Buchloe dactyloides Bulbilis dactyloides

Bursa bursa-pastoris Capsella bursa-pastoris

Calamagrostis $\mathrm{sp}$.

Cemelins sp.

Camelina dentata

Camelina microcarpa

Camelina sativa

Campe sp.

Campe barbarea

Barbarea barbarea

Barbarea vulgaris

Campe Verna

Barbarea verna

Barbarea praecox

Cannabis sativa

Capriola dactylon

Cynodon dactylon
Perennial quaking-gress: juak grass

Iittle quaking-grass

Bromegrass

Field brome; field cht

Rattlesnake cheso

California brome

Rescue grass

Hairy chess

Soft chess

Smooth brome; awnless brome grass

Smooth chess; bald chess

Ripgut grass

Chess; cheat

Barren chess; poverty chess

Downy chess; slender chess

Rescue grass

Buffalo grass

Shepherds-purse

Reedgrass

False-flax

Flatseed false-flax

Littleseed false flax

Bigseed false-flax

Wintercress

Bitter wintercress

Early wintercress

Hemp

Bermuda grass 


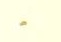


Capsella bursa-pastoris Bursa bursa-pastoris

Carduus sp.

Carduus arvensis

Cirsium arvense

Carduus crispus

Carduus discolor Cirsium discolor

Carduus lanceolatus Cirsium lanceolatum

Carduus undulatus Cirsium undulatum

Carex sp.

Carex franki i

Carthamus tinctorius

Carum oreganum

Cassia sp.

Cassia marilandica

Cassia nictitans

Cathartolinum virginianum

Linum virginianum

Caucalis nodosa

Torilis nodosa

Cenchrus sp.

Cenchrus pauciflorus

Cenchrus tribuloides

Centaurea sp.

Centaurea americana

Centaurea calcitrapa

Centaurea cyanus

Centaurea jacea

Centaurea melitensis

Centaurea picris

Centaurea solstitialis

Cephalaria transylvanica

Cerastium sp.
Shepherds-purse

Thistle

Canada thistle

Curly thistle

Field thistle

Buil thistle

Plains thistle

Sedge

No conmon name

Sefflower

Eppaw

No common name

Wild senne

Sensitive-pea

Woodland flax; wild yellow flox

Hedgeparsley; knotted hedge-parsley

Sendbur

Field sandbur

Dune sandbur

Star-thistle

Basketflower

Star-thistle

Cornflower

Brownscale knapweed

Malte ster-thistle

Russian knapweed

Yellow star-thistle; Barnaby's knapweed thistle

No cormon name

Cerastium 


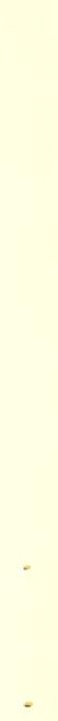


Cerastium $\nabla$ iscosum

Cerastium vulgatum

Chaetochloa italica Setaria italica

Chaetochloa lutescens Setaria lutescens

Chaetochloa viridis setaria viridis

Chaetochloa verticillata Setaria verticillata

Chaerophyllum procumbens

Chamaesyce preslii Euphorbia preslii Euphorbia nutans

\section{Chamaesyce maculate} Euphorbia maculata

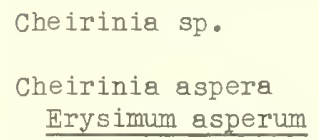

Sticky mouse-ear

Big mouse-ear; mouse-ear chickweed

Foxtail millet: German; Hungarian; golden (common); and others

Yellow bristlegrass; yellow foxtail

Green bristlegrass; green foxtail

Bur bristlegrass

No conmon name

Nodding spurge

Spotted spurge

Blistercress

Rough blistercress; orange mustard

Treacle-mustard; wormseed mustard

Goosefoot

Lamb squarters

Mexican-tea; wormseed

Pitseed goosefoot

No common name

Narrowleaf goosefoot

Nettleleaf goosefoot

Fingergrass

Australian fingergrass

Rhodes grass

Feather fingergrass

Skeletonweed

Oxeye daisy

Corn-marigold

Chicory 
Cicuta sp.

Cicuta maculata

Cirsium sp.

Cirsium arvense Carduus arvensis

Cirsium discolor Cerduus discolor

Cirsium lanceolatum Carduus lanceolatus

Cirsium undulatum Carduus undulatus

Claytonia perfoliata

Cleome serrulata

Clinopodium acinos Satureja acinos

Clinopodium vulgare

Cnicus benedictus

Collinsia sp.

Collomia grandiflora

Comptonia peregrina Comptonia asplenifolia Myrica asplenifolia

Conium meculatum

Conringia orientalis

Convolvalus sp.

Convolvalus arvensis

Convolvulus sepium

Coreopsis tinctoria

Coriandrum sativum

Corispermum hyssopifolium

Coronilla varia

Corynephorus canescens Weingartneria canescens

Crepis sp.

Crepis capillaris Crepis virens

Crepis setosa

Crepis tectorum

Crotalaria sp.
Waterhemlock

Spotted waterhemlock

Thistle

Canada thistle

Field thistle

Bull thistle

Plains thistle

Minerslettuce

Bee-flower; pink cleome

Basil-thyme

Wildbasil

Blessed thistle

No cormon name

No common name

Sweetfern

Poisonhemlock

Hares-ear-mustara

Bindweed

Bindweed; field bindweed; creeping jenny

Hedge bindweed

Calliopsis

Coriander

Tickseed; bug-seed

Crownvetch

Club-awn

Hawksbeard

Smooth hewksbeard

No common name

Narrowleaf hawksbeard

Crotalaria 


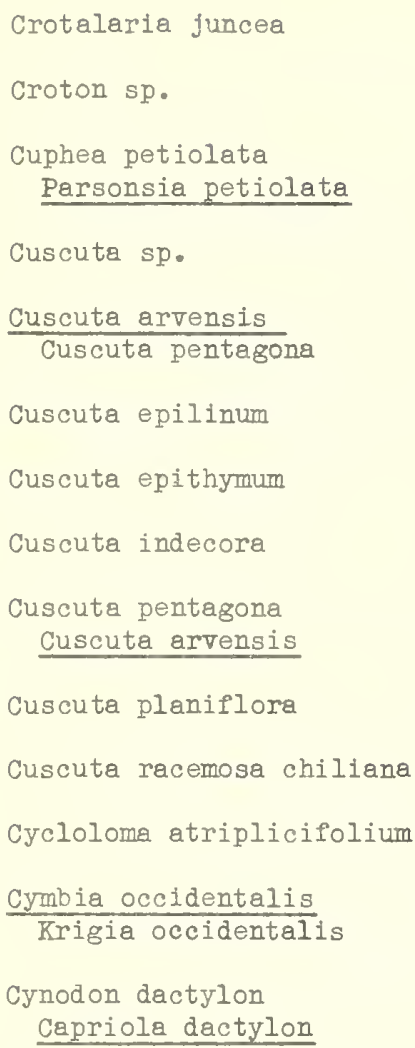

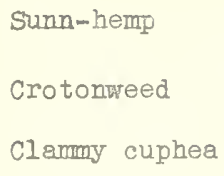

Wild carrot 
Delphinium consolida

Deschampsia sp.

Deschampsia caespitosa Aira caespitosa

Deschampsia flexuosa Aira flexuosa

Desmodium tortuosum Meibomia tortuosa Melbomia purpurea

Dianthus armeria

Digitalis purpurea

Digitaria sp.

Digitaria exilis Syntherisms exile

Digitaria filiformis Syntherisma filiformis

Digitaria ischaemum Syntherisma 1schaemum

\section{Digitaria orbata \\ Digitaria sanguinalis Syntherisma sanguinalis}

Diodia teres

Dipsacus sylvestris

Distichlis sp.

Distichlis spicata

Distichlis stricta

Dond la sp. Suaeda sp.

Downingia sp.

Dracocephalum sp. Moldavica sp.

Dracocephalum parviflorum Moldavica parviflora

Dracopis amplexicaulis Rudbeckla amplexicaulis

Dyssodia papposa Boebera papposa

Echinochloa colonum Panicum colonum

Echinochloa crusgalli
Field larkspur

Halrgrass

Tufted hairgrass

Crinkled halrgrass; eastern hairgrass

Florida beggarweed; beggarweed; Cherokee clover

Deptford pink

Common foxglove

Crabgrass

Fundi grass

Slender crabgrass

Smooth crabgrass; small crabgrass

No common name

Crabgrass

Rough buttonweed

Wild teasel

Saltgrass

Seashore saltgrass; marsh spikegrass

Desert saltgrass

Seepweed; sea blite

No cormon name

Dragonhead

American dragonhead

Clasping coneflower

Prairie-dog-weed

Jungle-rice

Barnyard grass 

$-12-$

Echinochloa crusgalli frumentacea Echinochloe frumentaces

Echium vulgare

Eleocharis sp.

Eleocharis obtusa

Eleocharis tenuis

Eleusine coracane

Eleusine indica

Elymus sp.

Epilobium sp.

Epilobium adenocaulon

Epilobium paniculatum

Eragrostis sp.

Eragrostis abyssinica

Eragrostis cilianensis Eragrostis major

Eragrostis poaeoides Eragrostis eragrostis Eragrostis minor

Erechtites hieracifolia

Erechtites prenanthoides

Eremochloa ophiuroydes

Erigeron sp.

Erigeron annuus

Erigeron canadensis Leptilon canadense

Erigeron strigosus Erigeron ramosus

Eriochloa sp.

Eriochloa punctata

Erodium cicutarium

Eruca sativa

Erucastrum gallicum Erucastrum pollichi

Ervum lens Lens esculenta Lentilla lens
Japanese millet

Blueweed

Spikerush

Blunt spikerush

slender spikerush

Ragi; African millet

Goosegrass; wiregrass; yardgrass

wild-rye

Willow-weed

sticky willow-weed

Panicle willow-weed

Lovegrass

Teff

Stinkgrass; strong-scented lovegrass

Little lovegrass

Burnweed; fire weed

Australian burnweed

Centipede grass

Fleabane

Whitetop fleabane

Hor seweed; Canada fleabane

Daisy fleabane

Cupgrass

Everlasting grass

Alfileria

Salad-rocket; rocquette

Rocket-weed; Pollicks mustard

Lentil 
$-13-$

Erysimum asperum

Cheirinia aspera

wrysimum cheirantholdes cheirinia cheiranthoides

Erysimum officinale Sisymbrium officinale

Eschscholtzla californica

Euphorbia sp.

Euphorbla cyparissias

Euphorbia esula

Euphorbia falcata

Euphorbia maculata Chamaesyce maculata

Euphorbia preslif Euphorbia nutans Chameesyce preslii

Euphrasia sp.

Fagopyrum vulgare Frgopyrum fagopyrum Fagopyrum esculentum

Festuca sp.

Festuca capillata Festuca tenuifolia

Festuca ovina duriuscula

Festuca elatior Festuca elatior pratensis

Festuca elatior arundinacea Festuca arundinacea

Festuca rubra heterophylla

F'estuca myuros

Festuca octoflora

Festuca ovina

Festuce rubra

Festuca rubra commutata Festuca rubra fallax

Festuca tenuifolia Festuca capillata

Fimbristylis autumnalis

Fimbristylis diphylla Fimbristylis laxe

Franseria discolor Gaertneria discolor
Rough blistercress; orange mustard

Treacle-mustard; wormseed mustard

Hedgemustard

California-poppy

Spurge

Cypress spurge

Leafy spurge

Sickle spurge

Spotted spurge

Nodding spurge

Eyebright

Common buckwheat

Fescue

Hair fescue

Hard fescue

Meadow fescue

Reed fescue

Shade fescue

Rattail fescue

Sixweeks fescue; slender fescue

Sheep fescue

Red fescue

Chewings fescue

Halr fescue

No cormon name

No cormon name

Skeletonleaf bur-sage; white-leaved gaertneria 
. 
Galega sp.

Galega officinalis

Galeopsis sp.

Galeopsis ladanum

Galeopsis tetrahit

Galinsoga parviflora

Galium sp.

Galium aparine

Gestridium lendigerum

Geranium sp.

Geranium carolinianum

Geranium columbinum

Geranium dissectum

Geranium molle

Geranium pusillum

Geum sp.

Geum canadense

Geum album

Gilia sp.

Gilia capitata

Glaucium corniculatum

Glyceria sp.

Glyceria fluitans Panicularia fluitans

Glyceria grendis Panicularia americana

Glyceria striata Panicularia nervata

Glycine hispida Soja $\max$

Glycyrrhiza lepidota

Godetia sp.

Gnaphalium sp.

Gnaphalium purpureum

Gnaphalium uliginosum

Grindelia sp.

Grindelia squarrosa
Goatsrue

Conmon goatsrue

Hempnettle

Red hempnettle

Hempnettle

Quickweed

Bedstraw

Catchweed; cleavers

Nitgrass

Cranesbill

Carolina cranesbill

Longstalk cranesbill

Cutleaf cranesbill

Dovefoot cranesbill

Sinall cranesbill

Avens

White avens

Gilia

Globe gilia

Sea-poppy

Mannagrass

Mannagrass

American mannagrass; tall mannagrass

Fowl mannagrass

Soybean

Wild licorice

Godetia

Cudweed

No common name

Mouse-ear cudweed; low cudweed

Gumweed

Curlycup gumweed 


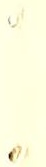

. 


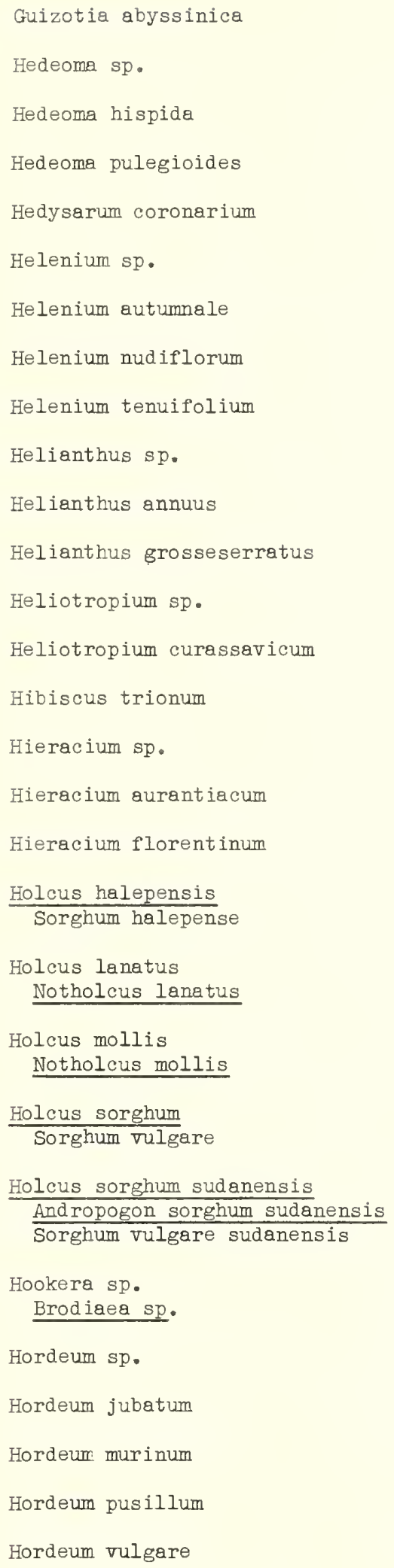


$-16-$

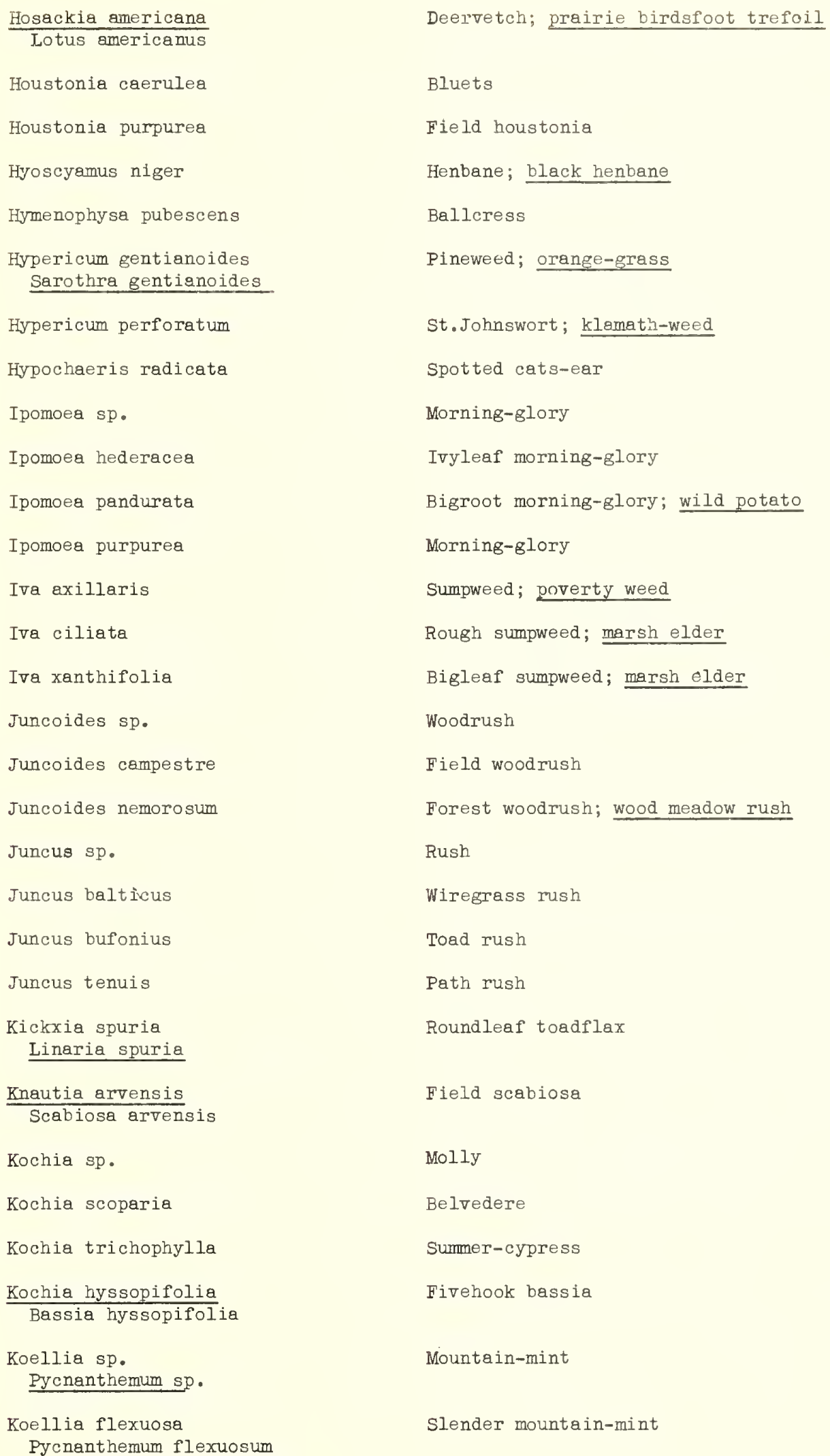




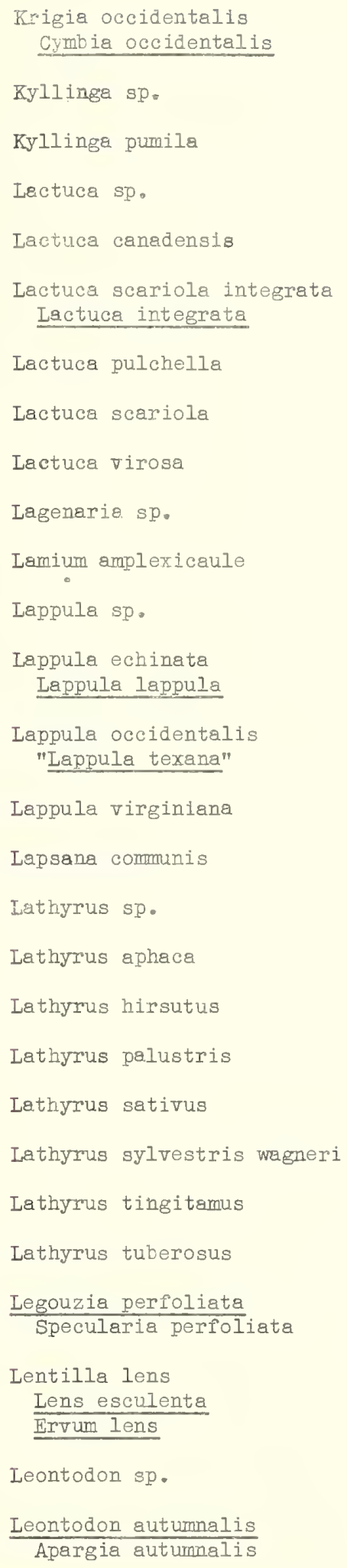

No common name

No common name

No cormon name

Lettuce

Tall lettuce

Prickly lettuce

Chicory lettuce

No conmon name

No common name

Gourd

Henbit; dead nettle

Stickseed

European stickseed; sticktight

Western stickseed; hairy stickseed

Virginis stickseed

Nipplewort

Pea

Yellow pea

Rough pea; perennial sweet pea

Marsh pea

Grass pea

Flat pea

Tangier pea

Groundnut per; vetchling

Venus lookingglass

Lentil

Dandelion

Fall-dandelion 
$-18-$

Leontodon laevigatum

Redseed dandelion

Taraxacum erythrospermum

Leontodon nudicaulis

Apargia nudicaulis

Leontodon taraxacum

Taraxacum officinale

Leonurus cardiaca

Lepidium sp.

Lepidium bipinnatifidum

Lepidium campestre

Lepidium densiflorum

"Lepidium apetalum"

Lepidium draba

Lepidium nitidum

Lepidium perfoliatum

Lepidium repens

Lepidium virginicum

Leptilon canadense

Erigeron canadens is

Leptochloa sp.

Leptochloa imbricata

Leptochloa uninervia

Lespedeza sp.

Lespedeza sericea

Lespedeza stipulacea

Lespedeza striata

Lespedeza striata var.

Linaria sp.

Linaria spuria Kickxia spuria

Linaria vulgaris Linaria linaria

Linum usitatissimum

Linum virginianum Cathartolinum virginianum

Lithospermum sp.

Lithospermum aryense

Rough hawkbit

Dandelion

Motherwort

Peppergrass

Wayside peppergrass

Field peppergrass; field cress

Prairie peppergrass

Perennial peppergrass; hoary cress; whiteweed

Tongue peppergrass

Clasping peppergrass

Lens peppergrass

American peppergrass

Horseweed; Canada fleabane

Sprangletop

No common name

Lespedeza

Chinese lespedeza; perennial lespedeza

Korean lespedeza

Common lespedeza

Kobe lespedeza

Toadflax

Roundleaf toadflax

Butter-and-eggs; common toadflax

Flax

Woodland flax; wild yellow flax

Gromwell

Field gromwell; corn gromwell 

$-19-$

Lobelia sp.

Lobelia inflata

Lolium sp.

Lolium multiflorum Lolium italicum

Lolium perenne

Lolium temulentum

Lolium rigidum duthiei

Lotus americanus Hosackia americana

Lotus corniculatus

Ludwigia alternifolia

Lupinus sp.

Lychnis $\mathrm{sp}$.

Lychnis alba

Lycopus virginicus

Lysimachia nummularia

Lythrum hyssopifolia

Madia glomerata

Madia sativa

Malva sp.

Malva crispa

Malva parviflora

Malva rotundifolia

Malva sylvestris

Malva verticillata

Marrubium vulgare

Matricaria chamomila

Matricaria inodora

Matricaria matricarioides

Medicago arabica

Medicago hispida Medicago hispida denticulata

Medicago lupulina

Medicago orbicularis
Lobelia

Indian-tobacco

Ryegrass

Italian ryegrass

Perennial ryegrass; English ryegrass

Darnel

No common name

Deervetch; prairie birdsfoot trefoil

Birdsfoot trefoil

Seedbox

Lupine

Campion

Evening campion; white campion

American bugleweed

Moneywort

Hyssop loosestrife

Cluster tarweed

Chilean tarweed

Mallow

Curly mallow

Little mallow

Running mallow; cheeses

High mallow

cluster mallow

White hoarhound

Wild-camomile; German false-camomile

False-camomile; scentless false-camomile

Pineappleweed

Spotted bur-clover

Californit bur-clover

Black medick; yellow trefoil

Buttonclover; kutton bur-clover 
) 


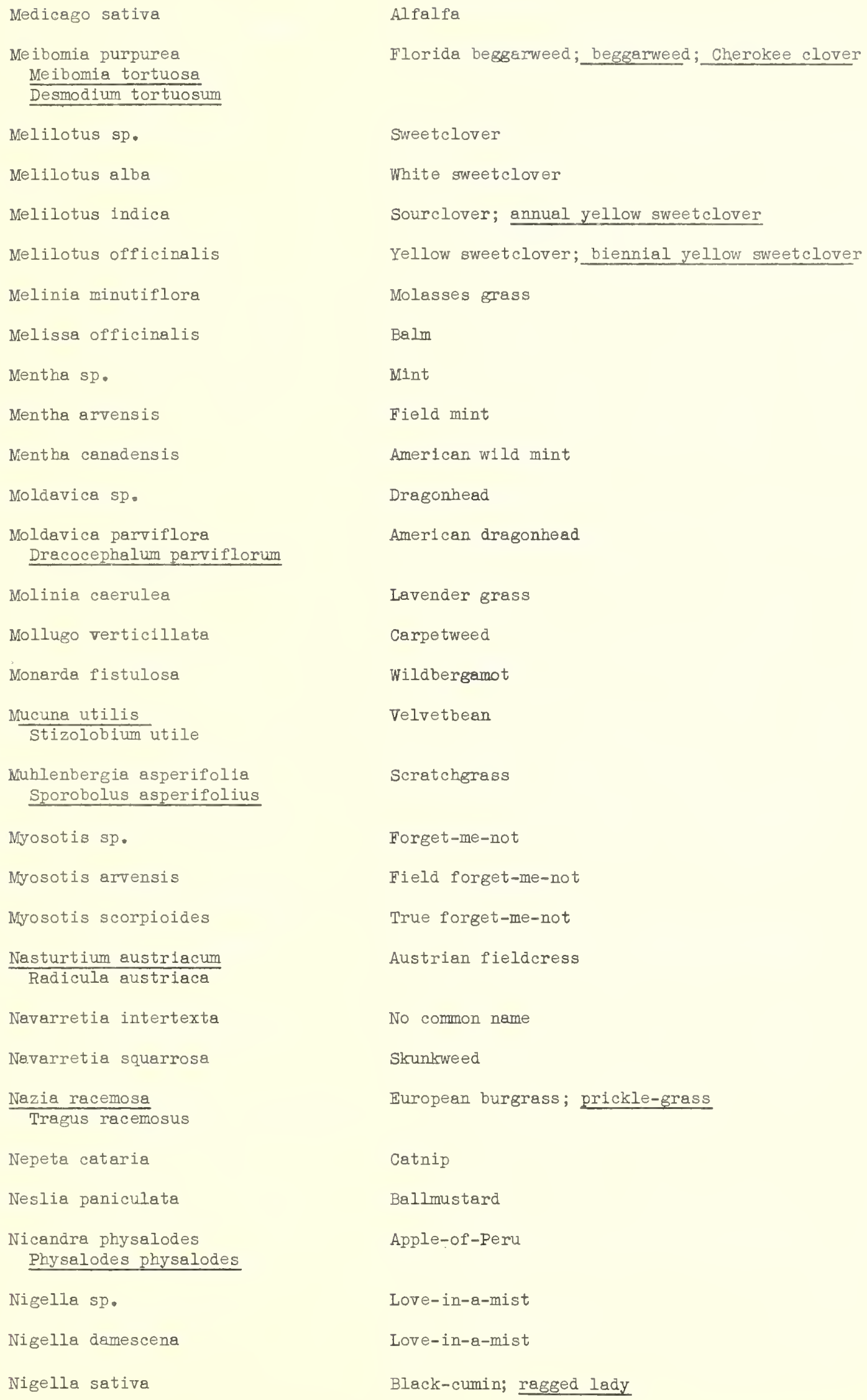



Norte altissima
Sisymbrium altissimum

Notholcus lanatus Holcus lanatus

Notholcus mollis Holcus mollis

Oenothera sp.

Denothera biennis

Onegra biennis

Oenothera laciniata Raimannia laciniata

Onobrychis viciaefolia Onobrychis vulgeris onobrychis sativa

onopordon acanthium

ornithopus sativus

Orobanche sp.

Orobanche ramosa

Oryza sativa

Oryzopsis sp.

Oryzopsis hymenoides

Oryzopsis miliacea

Osmorhiza longistylis

oxalis stricta

Pantcularia americana Glyceria grandis

Panicularia fluitans Glyceria fluitans

Panicularia nervata Glyceria striata

Panicum anceps Panicum rostratum

Panicum capillare

Panicum capillare occidentale Panicum barbipulvinatum

Panicum colonum Echinochloa colonum

Panicum dichotomiflorum

Panicum fasciculatum Panicum fuscum

Panicum gracile
Tumblemustard

Velvet grass

Softgrass; meadow soft grass; German mesquite grass

Evening-primrose

Evening-primrose

Cutleaf evening-primrose

Sainfoin

Cottonthistle; Scotch thistle

Serradella

Broomrape

Hemp broomrape

Rice

Ricegrass

Indian ricegrass; quincy grass

No common name

Aniseroot; sweet cicely

Yellow oxalis; yellow wood sorrel

American mannagrass; tall mannagrass

Mannagrass

Fow I mannagrass; nerved mannagrass

No common name

Witchgrass

No common name

Jungle-rice

Fall panicum; spreading panicum

Browntop millet

No common name 

Panicum hians

Steinchisma hians

Panicum hillmani

Panicum lanuginosum

Pantcum maximum

Panicum miliaceum

Panicum purpurascens

Panicum rostratum Panicum anceps

Panicum virgatum

Papaver sp.

Papaver dubium

Papaver rhoeas

Papaver somniferum

Parsonsia petiolata Cuphea petiolata

Paspalum boscianum

Paspalum compressum Axonopus compressus

Paspalum dilatatum

Paspalum distichum

Paspalum laeve

Paspalum notatum

Paspalum setaceum

Paspalum urvillei Paspalum larranagai

Pastinaca sativa

Pedicularis sp.

Pennisetum glaucum Pennisetum spicatum

Pennisetum purpureum

Petalostemum candidum

Phacelia sp.

Phalaris sp.

Phalaris angusta

Phalaris arundinacea
No common name

No common name

No common name

Guinee grass

Proso; broomcorn millet; hog millet

Para grass

No common name

Switchgrass; tall smooth panicum

Poppy

Longpod poppy

Corn poppy

Optum poppy

clammy cuphea

Bull paspalum

Carpet grass

Dalıis grass

Knotgrass

Field paspalum

Bahia grass

Slender paspalum

Vasey grass

Parsnip

Fernleaf

Pearl millet

Napier grass; merker grass

White prairieclover

Curlybloom

Canary grass

Timothy canary grass

Reed canary grass 



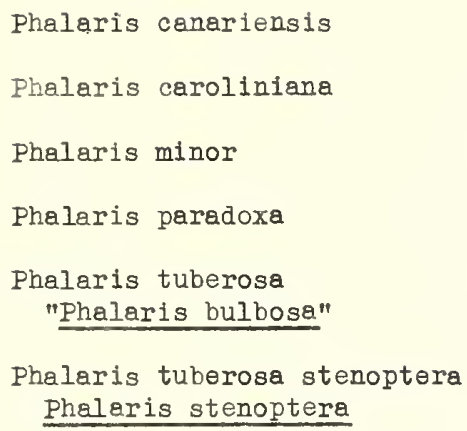

Canary grass

Carolina canary grass

Little canary grass

Hidehead canary grass; gnawed canary grass

No cormon name

Harding grass; Peruvian winter grass

Timothy

Ground cherry

Round leaf ground cherry

Apple-of-Peru

Pokeberry

Oxtongue

Hawkweed picris

Pea

Field pea

Garden pea

Branching plantain

Bottlebrush plantain; bracted plantain

Crowfoot plantain

No common name

Buckhorn plantain

Rippleseed plantain

Spogel plantain; blond psyllium

Fleaseed plantain; psyllium

Woolly Indianwheat

Redseed plantain

Blackseed plantain

Paleseed plantain; dwarf plantain

Bluegrass

Annual bluegrass 


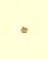


Poa arachnifera

Poa bulbosa

Poa compressa

Poa nemoralis

Poa palustris

Poa triflora

Poa pratensis

Poa sudetica

Poa trivialis

Poa triflora

Poa palustris

Polygala sp.

Polygonum sp.

Polygonum argyrocoleon

Polygonum aviculare

Polygonum convolvulus

Polygonum hydropiper

Polygonum kelloggii

Polygonum lapathifolium

Polygonum muhlenbergil

Polygonum emersum

Polygonum pensylvanicum

Polygonum persicaria

Polygonum punctatum

Polypogon monspeliensis

Portulaca oleracea

Potentilla sp.

Potentilla biennis

Potentilla canadensis

Potentilla monspeliensis

Potentilla rivalis

Prunella vulgaris

Psoralea sp.

Psoralea tenuifolia

Puccinellia nuttalliana

Pycnanthemum sp.

Koellia sp.
Texas bluegrass

Bulbous bluegrass

Canada bluegrass

Wood bluegrass; wood meadow grass

Fowl bluegrass

Kentucky bluegrass

No common name

Rough bluegrass; rough-stalked meadow grass

Fowl bluegress

Polygala

No common name

Silversheath knotweed

Knotweed

Cornbind; black bindweed

smartweed

No cormon name

Pole ledysthumb; pale knotweed

Bigroot ladysthumb; water smartweed

Bigseed ladysthumb; Pennsylvania knotweed

Ladysthumb

American smartweed

Rabbitfoot grass

Purslane

Cinquefoil

No common name

oldfield cinquefoll; five finger

Strawberryweed; cinquefoil

No common name

Selfheal

Scurf-pea

No conmon name

Nuttall alkali-grass; zwadke alkali-grass

Mountain-mint 


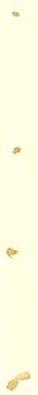

. 
Pycnanthemum flexuosum Koellia flexuosa

Radicula sp. Roripe sp.

Radicula austriaca Nesturtium austriacum

Raimannia laciniata Oenothera laciniata

Ranunculus sp.

Ranunculus abortivus

Ranunculus acris

Ranunculus arvensis

Ranunculus bulbosus

Ranunculus parviflorus

Ranunculus repens

Ranunculus sardous Ranunculus parvulus

Renunculus sceleratus

Raphenus raphanistrum

Raphanus sativus

Reseda lutea

Reseda odorata

Richardia scabra Richardsonia scabra

Ricinus communis

Rosa sp.

Rosa blanda

Roubieva multifida

Rudbeckia sp。

Rudbeckia emplexicaulis Dracopis amplexicaulis

Rudbeckia hirte

Rubus sp.

Rumex sp.

Rumex acetosa

Rumex acetosella

Rumex conglomeratus

Rumex crispus
Slender mountain-mint

Marshcress

Austrian fieldcress

Cutleaf evening-primrose

Buttercup

Littleleaf crowfoot

Tall buttercup

Corn crowioot

Bulb buttercup

Sticktlght crowfoot; sinall-flowered buttercup

Creeping buttercup

Wartseed buttercup; hairy buttercup

Blister crowfoot; celery-leaved crowfoot

Wild radish

Radish

Base-rocket; mignonette

Mignonette

Mexican-clover

Castor-bean

Rose

Meadow rose

Flatweed

Coneflower

Clasping coneflower

Black-eyed-susan

Blackberry

No common name

Garden sorrel; sour dock

Sheep sorrel

Green dock

Curly dock 



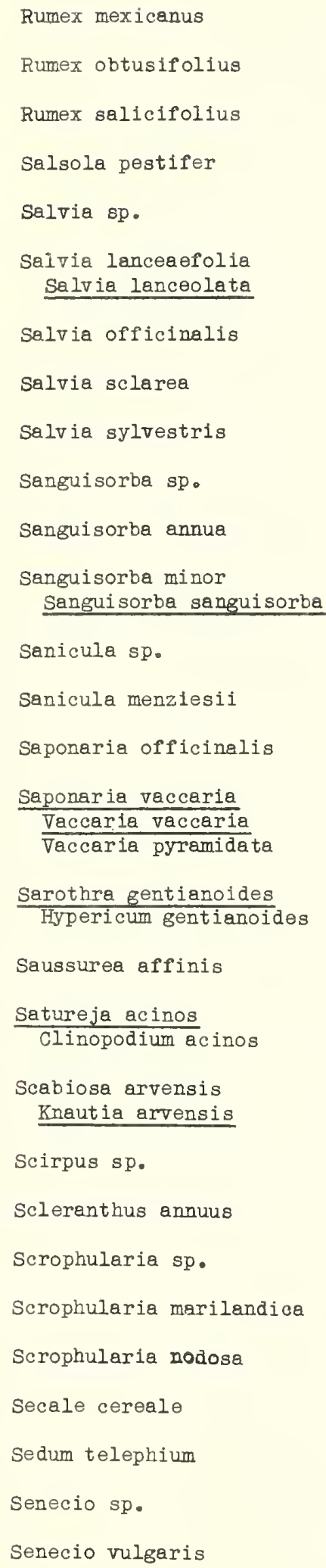

No common neme

Bitter dock

No cormon name

Russian-thistle

Sage

Lanceleaf sage

Garden sage

Clary; clear-eye

Pasture sage; wild sage

Burnet

Prairie burnet

Little burnet

Saniole

Foothill senicle

Bounc ing-bet

Cowcockle

Pineweed; orange-grass

No common name

Basil-thyme

Field scabiosa

Bulrush

Knawel; German knotgrass

Figwort

American figwort; Maryland figwort

Wood figwort

Rye

Liveforever

Groundsel

Ground sel 



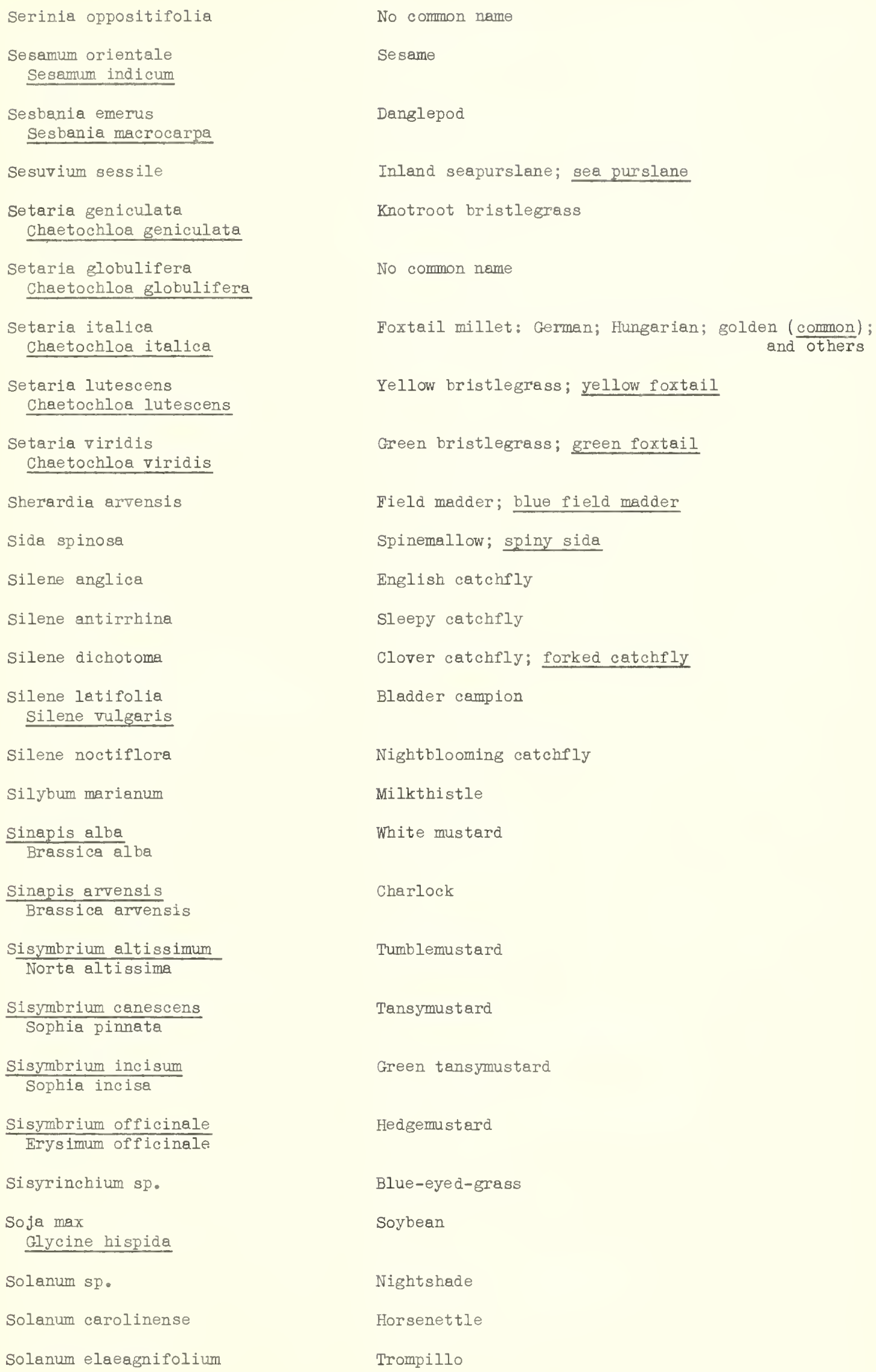

Serinia oppositifolia

Sesamum orientale Sesemum indicum

Sesbania emerus Sesbania macrocarpa

sesuvium sessile

Setaria geniculata Chaetochloa geniculata

Setaria globulifera Chaetochloa globulifera

Setaria italica Chaetochloa italica

Setaria lutescens Chaetochloa lutescens

Setaria viridis Chaetochloa viridis

Sherardia arvensis

Sida spinosa

Silene anglica

Silene antirrhina

Silene dichotoma

Silene latifolia Silene vulgaris

Silene noctiflora

Silybum marianum

Sinapis alba Brassice alba

Sinapis arvensis Brassica arvensis

Sisymbrium altissimum Norta altissime

Sisymbrium canescens Sophia pinnate

Sisymbrium incisum Sophia incisa

Sisymbrium officinale Erysimum officinale

Sisyrinchium sp.

Soja $\max$ Glycine hispida

Solanum sp.

Solanum carolinense

Solanum elaeagnifolium

No cominon name

Se same

Danglepod

Inland seapurslane; sea purslane

Knotroot bristlegrass

No common name

Foxtail millet: German; Hungarian; golden (common); and others

Yellow bristlegrass; yellow foxtail

Green bristlegrass; green foxtail

Field madder; blue field madder

Spinemallow; spiny sida

English catchfly

Sleepy catchfly

Clover catchfly; forked catchfly

Bladder campion

Nightblooming catchely

Milkthistle

White mustard

Charlock

Tumblemustard

Tansymustard

Green tansymustard

Hedgemu stard

Blue-eyed-grass

Soybean

Nightshade

Horsenettle

Trompillo 

Solenum nigrum

Solanum rostratum

Solanum triflorum

Solidego $\mathrm{sp}$.

Solidago canadensis

Sonchus sp.

Sonchus arvensis

Sonchus asper

Sonchus oleraceus

Sophia pinnata

Sisymbrium canescens

Sophia incisa

Sisymbrium incisum

Sorghum halepense

Folcus halepensis

Sorghum virgatum

Sorghum vulgare

Holcus sorghum

Sorghum vulgare sudanense

Holcus sorghum sudanensis

Andropogon sorghum sudanens is

Specularia perfoliata

Legouzia perfoliata

Spergula arvensis

Spergula pentandra

Spergularia rubra

Tissa rubra

Sporobolus sp.

Sporobolus asperifolius Muhlenbergia esperifolia

Sporobolus clandestinus

Sporobolus cryptandrus

Sporobolus neglectus

Sporobolus rigens

Stachys sp.

Stachys agraria

Stachys annua

Stachys palustris

Steinchisma hians

Panicum hians
Black nightshede

Buffalo-bur

Cutleaf nightshade

Goldenrod

Canada goldenrod

Sowthistle

Perennial sowthistle

Prickly sowthistle; spiny sowthistle

Common sowthistle

Tansymustard

Green tansymustard

Johnson grass

Tunis grass

Sorghum

Sudan grass

Venus lookingglass

Corn spurry

Wingseed spurry

Red sandspurry

Dropseed

Scratchgrass

Scratch dropseed; rush grass

Sand dropseed

Puff dropseed; small mash grass

No common name

Betony

Shade betony

Field betony; hedge nettle

Marsh betony; hedge nettle

No common name 

Stellaria eraminea Alsine gramineg

Stellaria media Alsine media

Stenophragma thallana Arabidopsis thaliana

Stipa $s p$.

Stipa cometa

Stipe viridula

Stizolobium utile Mucune utilis

Swainsona salsula

Syntherismg sp.

Syntherisms exile Digitaria exilis

Syntherisma filiformis Dlgitaria filiformis

Syntherisme ischaemum Digitaria 1schaemum

Syntherisma sanguinalis Digitaria sanguinalis

Tarexacum erythrospermum Leontodon laevigatum

Taraxacum officinale Leontodon taraxacum

Tecoms radicans Blgnonia radicans

Teucrium sp.

Teucrium canadense

Teucrium scorodonla

Thalictrum sp.

Thlaspi arvense

Thymus serpyllum

Tissa sp.

Tissa rubra Spergularia rubra

Torilis nodosa Caucalis nodose.

Tragopogon porrifolius

Tragopogon pratensis

Tragus racemosus Nazia racemosa
Little starwort

Chickweed

Mouse-ear-cress

Needlegress

Needle-and-thread; western st1pa; feathergrass

Green needlegrass; feathergrass

Velvetbean

No cormon name

Crabgrass

Fundi grass

Slender crabgrass; slender flnger-grass

Smooth crabgrass

Crabgrass

Redseed dandellon

Dendelion

Trumpetcreeper

Germander

American germander

Wood germander

Meadowrue

Pennycress; Frenchweed

Mother-of-thyme

Sandspurry

Red sandspurry

Hedgeparsley; knotted hedge parsley

Oysterplant; vegetable-oyster

Buckbeerd

European burgress; prickle-grass 


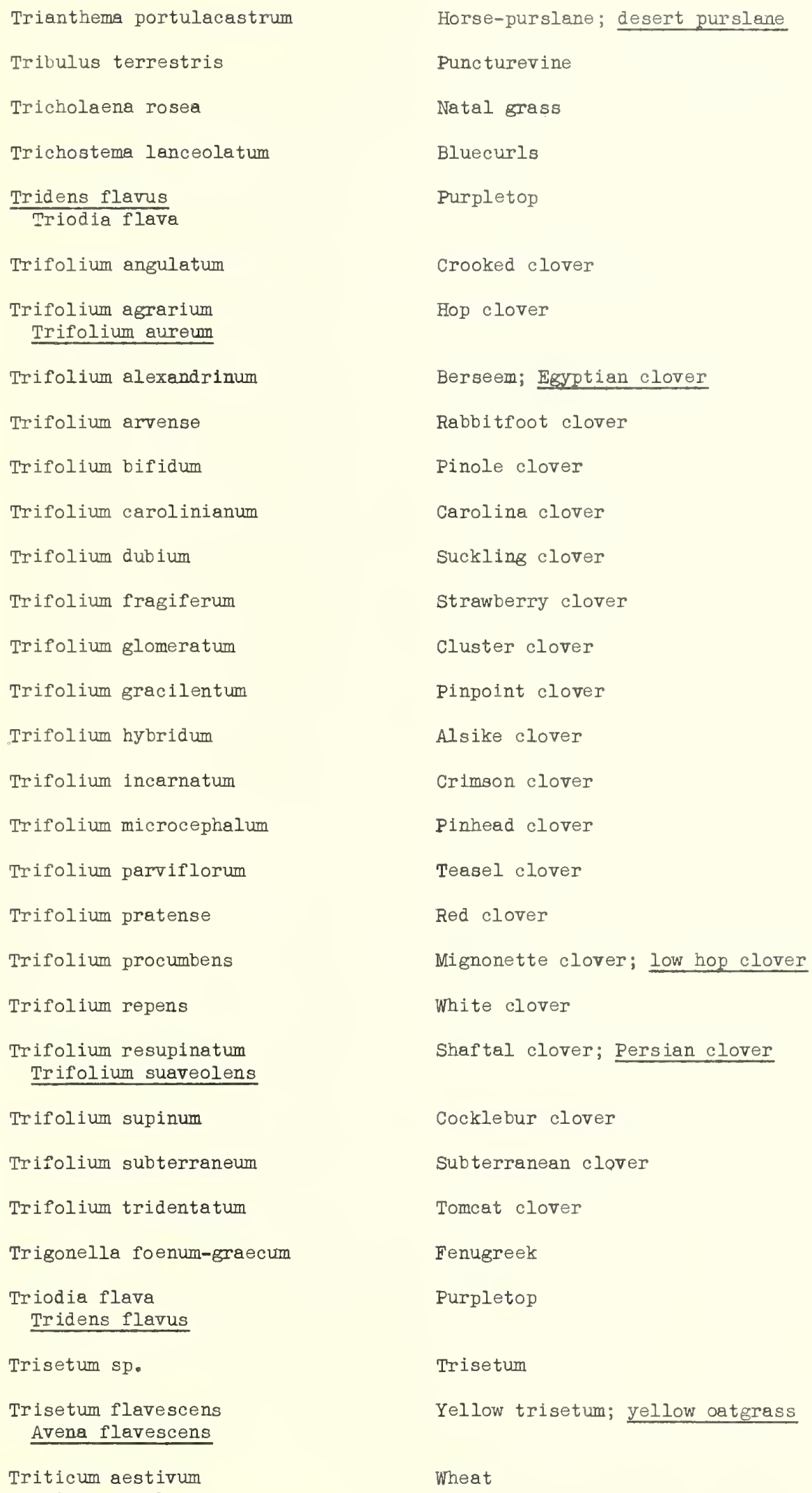


Urtica sp.

Urtica dioica

Urtica gracilis

Vaccaria pyramidata

Vaccaria vaccaria

Seponaria vaccaria

Valerianella sp.

Valerianella dentata

Valerianella eriocarpa

Valerianella locusta

Verbescum sp.

Verbascum blattaria

Verbascum phlomoides

Verbascum thapsus

Verbena sp.

Verbena angustifolia

Verbena hastata

Verbene officinalis

Verbena stricta

Verbena urticifolia

Vernonia sp.

Vernonia noveboracens $1 \mathrm{~s}$

Veronica sp.

Veronica agrestis

Veronica arvensis

Veronica hederaefolia

Veronica officinalis

Veronica peregrina

Veronica serpyllifolia

Vicia sp.

Vicla angustifolia

Vicia atropurpurea

Vicia cracca

Vicia dasycarpa

Vicla ervilia
Nettle

Big nettle; stinging nettle

Narrowleaf nettle; slender nettle

Cowcockle

Cornsalad

Toothed cornsalad

Italian cornsalad

Cornsalad

Mulle 1n

Moth mullein

Clasping mullein

Flannel mullein

Vervain

Narrowleaf vervain

Blue vervain

Vervain

Hoary vervain

White vervain

Ironweed

Coest ironweed

Speedwell

Field speedwell

Corn speedwe11

Ivyleaf speedwell

Male speedwell

Purslane speedwell

Thymeleaf speedwell

Vetch

Narrowleaf vetch

Purple vetch

Bird vetch

Woollypod vetch

Bitter vetch 

Vicia faba

V1012 gracilis

Vicie grendiflore

Vleje hirsute

Vicia Iutea

Victe monentha

Vicia pannonica

Vicia sativa

Vicia tetresperma

Vieia villosa

Vigna sinensis

Viola tricolor

Weingartneria canescens Corynephorus cenescens

Xanthium sp.

Janthium spinosum.

Zoyala Japonica

Zoyaia matrella Zoysta pungens

Zoysia temuifolia Osterdamia tenuifolia
Broadbean

Slender vetch

No common name

Tiny vetch

Yellow vetch

Monenthe vetch

Hungerian vetch

Common vetch

Fourgeed vetch

Halry retch

Cowper

Pansy

Club-amn

Cocklebur

spiny cocklebur

Japenese lawn grass

Manila grasB

Mascarene grass 
N 


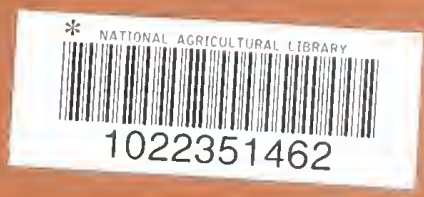


